\title{
Club Characteristic and the Proximity Effect of Travel Links between Cities in China
}

\author{
Mingxing Chen, ${ }^{1,2}$ Xinyue Luo, ${ }^{1,2}$ Maogui $\mathrm{Hu}^{3}$ and Xiaoping Zhang $\mathbb{D}^{2}$ \\ ${ }^{1}$ Institute of Geographic Science and Natural Resources Research, Key Laboratory of Regional Sustainable Development Modeling, \\ CAS, Beijing 100101, China \\ ${ }^{2}$ College of Resources and Environment, University of Chinese Academy of Sciences, Beijing 100049, China \\ ${ }^{3}$ Institute of Geographic Science and Natural Resources Research, \\ State Key Laboratory of Resources and Environmental Information System, CAS, Beijing 100101, China
}

Correspondence should be addressed to Xiaoping Zhang; zhangxp@ucas.ac.cn

Received 14 May 2020; Revised 5 July 2020; Accepted 20 July 2020; Published 25 August 2020

Guest Editor: Jun Yang

Copyright (C) 2020 Mingxing Chen et al. This is an open access article distributed under the Creative Commons Attribution License, which permits unrestricted use, distribution, and reproduction in any medium, provided the original work is properly cited.

\begin{abstract}
With the increasingly close relations between cities in China, it is of great significance to explore the regular characteristics of the intercity connection. Through Tencent's population migration heat and Baidu map big data, this paper analyzes the regular characteristics of the relations between complex cities based on such index as the rich node propensity index, preference level index, and relative heat index and also investigates the influence of geographical proximity factors on the external relations of different cities. The research has the following results. Firstly, the relations between cities have obvious club characteristics. The rich nodes tend to connect with the rich nodes, while the nonrich nodes tend to connect with the nonrich nodes. Secondly, the connection between cities has the effect of hierarchical proximity, and cities mainly establish spatial connections with cities of the same level and adjacent level. Thirdly, the relations between cities also have the effect of geographical proximity, and the degree of influence of geographical proximity in low-level cities is greater than that in high-level cities. Fourthly, the external connection mode of high-level cities is to establish close contact with high-level cities adjacent to the level, with strong attraction to low-level cities adjacent to the location at the same time. The low-level cities are closely related to the high-level cities adjacent to the location and other cities of geographical proximity or adjacent level. This study helps to further understand the complex characteristics and laws of intercity connections and urban networks.
\end{abstract}

\section{Introduction}

With the rapid development of China's economy and society and the rapid promotion of urbanization [1, 2], cities are increasingly closely connected in China. The rapid construction of high-speed railway, expressway, and other transportation networks provides convenient conditions for the flow of population, capital, information, technology, and other elements between cities $[3,4]$. Urban development is no longer confined within the city, and administrative barriers are gradually broken. China's urban system is changing from hierarchical to a flat network. Since the 21 st century, with China's city linkages and city network evolving, although a few center cities still occupy the absolute controlling position, but the city link is no longer limited to between core cities and between core cities and general cities, connection between general cities gradually enhances and the connectivity of the urban network is increasing. At a critical time of growing intercity connections, it is of great practical significance to explore the characteristics of spatial connection between cities in China so as to understand the operation mechanism behind intercity connections. Academic circles focus on researches on the relations between cities and the complexity of the urban network [5]. Yet, there is still a lot of space to explore in the research of revealing the characteristics of urban interaction and mining its regular characteristics and mechanisms. 
In the recent years, with the construction of Internet infrastructure and the popularization of smart phones, the number of Internet users in China is increasing rapidly. According to the economic operation of the communication industry in August released by the Ministry of Industry and Information Technology of the People's Republic of China, China's mobile Internet users reached 1.004 billion in 2018. Tencent is the main service provider of China's communication social applications, and its rich mobile products attract a large number of users. Tencent products position more than 10 billion times per day. The company has released Tencent population migration heat data, the intercity population flow data which is calculated on the basis of mobile phone positioning data updating every five minutes. These data accurately reflect the real-time travel situation of the population between two cities in China and can represent the relations between China's cities, which better meets the research needs of this paper. The data have been used to discuss the network characteristics $[6,7]$, but the characteristics and patterns of intercity connections deserve further study.

This paper grabs the data of population migration heat per five minutes of Chinese cities published by Tencent from January 1 to December 31 in 2018, calculates the average value of migration heat between two cities every day, and then, sums up the data of 365 days in the whole year to get the data of population travel heat between cities in 2018, which is set as the data basis of this research. This paper mainly studies the characteristics of intercity relations in China, such as club characteristics and geographical proximity effect, including six parts. The first part is the introduction. The second part reviews the research progress of urban connection and urban network. The third part introduces the research methods and data sources. The fourth part gives the research results from three aspects: the general characteristics, the rich node propensity and the hierarchical proximity effect, and the geographical proximity effect of intercity connections. The fifth part further analyzes the typical characteristics and modes of intercity relations, including club characteristics, geographical proximity and hierarchical proximity effects, and the spatial distance threshold effect. Finally, the main conclusions and contribution of this paper are summarized briefly. This study maps the relations between China's cities through the population travel between cities and explores the characteristics of preferences in various aspects of urban external relations, as well as differences of different cities, which helps to reveal and understand the complexity law and operation mechanism of urban spatial relations.

\section{Review of the Related Literature}

The perspectives of urban connection and urban network research are diverse. In the past, scholars often used the data of communication and express $[8,9]$, intercity traffic shift [10-14] and the number of distribution of parent companies and subsidiary companies [15, 16], social contact [17], and knowledge "flow" [18] to describe the relations between cities. Since people bring the vitality of urban development, the flow of population between cities also reflects the connection between cities. However, because of the difficulty to directly obtain the population "flow" data under urban scale, the past research mostly used statistical data to measure the intercity connection under the perspective of population flow $[19,20]$. Nevertheless, this method often has data bias. In some studies, the intensity of population mobility between cities is estimated by the gravity model to represent the intercity connection; that is, it is assumed that population mobility is in direct proportion to the economic level of the origin and destination and inversely proportional to the distance between the two places [21, 22]. This approach is scientific and feasible to a certain extent, but it is still not accurate enough to express the connections between cities.

Recently, with the rapid development of big data calculation and processing methods, it is possible to completely and accurately depict the population flow between cities. For example, Tencent in China releases the big data of Tencent population migration heat, which express the real-time situation of population movement between cities. Baidu also releases similar data. Big data overcome the shortcomings of traditional data to some extent [23, 24], reflecting more accurate population movement in real time. Some scholars have made use of big data of population mobility to carry out researches on the intercity connection and urban network [6, 7, 25-29]. For example, the paper uses Baidu migration big data to study the characteristics of the rich club of the population mobility network during the Spring Festival travel rush in China [30] and the multisource migration big data of Baidu, Tencent, and Qihoo to study the travel characteristics of the population during the Spring Festival travel rush in China [25]. Big data provide a richer and more accurate data foundation for the study of urban connection and urban network, which enables the related research to be explored in depth from many aspects.

The related researches on intercity connection mainly focus on the geographical characteristics of intercity connection [6, 25, 31, 32] and urban network topology [33-37]. Yet, only a few studies have analyzed the characteristics of intercity connection [38, 39], such as the phenomenon of rich club of intercity linkages [40-42]. Researches show that urban network has a typical feature of rich club [43-45], that is, there is a trend of rich nodes preferentially connecting to rich nodes in the urban network. Although there has been a considerable amount of research in this area, many aspects need to be further explored. For example, the current literature only depicts the rich club characteristics of the network while ignores the connection characteristics of nonrich nodes. At the same time, some studies have determined that nodes prefer to connect rich nodes or nonrich nodes [30], lacking quantitative description of the degree of preference between nodes and rich nodes. In addition, geographical proximity plays an important role in the connection between cities [46]. For different cities, it is worth exploring whether there are differences in the impact of geographical proximity.

Based on the progress and lack of related researches, it is necessary to carry out this study. The purpose of this paper is 
to investigate the preference characteristics of urban connections in China, including rich nodes (high-level cities) and nonrich nodes (low-level cities) and quantify the preference degree of nodes to connect with rich nodes or nonrich nodes. At the same time, the paper explores the influence of geographical proximity on urban connection in China and analyzes the difference of the influence of geographical proximity on different cities.

\section{Research Methods and Data Sources}

3.1. Data Availability. This paper grabs the data of population migration heat per five minutes of Chinese cities published by Tencent from January 1 to December 31 in 2018, calculates the average value of migration heat between two cities every day, and then, sums up the data of 365 days in the whole year to get the data of population travel heat between cities in 2018, which is set as the data basis of this research on the characteristics of intercity connection. Tencent's population migration heat data is obtained from the location big data released by the Tencent company, which come from https://heat.qq.com/. Besides, the shortest travel time data between cities are obtained from the Baidu map product released by the Baidu company, which come from https://map.baidu.com/. The Baidu map platform provides people with enquiry of travel time and route under three modes of transportation, such as trains, planes, and automobiles. This paper compares the travel time of three modes of transportation between two cities in China and, then, selects the shortest time as the shortest travel time between cities.

3.2. Rich Rode Propensity Index. Some studies show that most nodes in China's urban network prefer to connect to rich nodes rather than nonrich nodes [30]. However, few studies quantitatively measure the degree of node preferring to connect rich nodes in China's urban networks. This paper proposes an index $P_{i}$ to measure the rich nodes propensity of network nodes.

$$
P_{i}=\sum_{j=1}^{367} \frac{\text { Heat }_{i j}}{\text { Heat_departure }_{i}} \times L_{j} .
$$

In the formula, $P_{i}$ is the rich node propensity index of node $i$, which means the degree of node $i$ preferring to connect rich nodes. Since $1<P_{i}<5$, the larger the value of $P_{i}$ is, the more intense the preference of node $i$ connecting rich nodes will be. Heat ${ }_{i j}$ is the travel heat between node $i$ and node $j$. Heat_departure ${ }_{i}$ is the departure heat of node $i$, that is, the sum of travel heat departing from node $i$ to other cities. $L_{j}$ is the rich node index of node $j$, which means the attributes of rich nodes of connected node $j$. The larger the value is, the more prominent the rich node attribute of the node $j$. According to the total heat of urban travel ${ }^{1}$, cities (nodes) are divided into five levels in the light of the classification of natural fracture point. When node $j$ belongs to the first level, $L_{j}=5$. When node $j$ belongs to the second level, $L_{j}=4$. When node $j$ belongs to the third level, $L_{j}=3$.
When node $j$ belongs to the fourth level, $L_{j}=2$. When node $j$ belongs to the fifth level, $L_{j}=1$. Heat $_{i j} /$ Heat $\_$departure ${ }_{i}$ represents the relative heat between node $i$ and node $j$ under the level of the external contact of node $i$. The larger value means the greater relative connection between node $i$ and node $j$; that is, node $i$ more prefers to establish connection with node $j$. The use of relative heat rather than absolute heat is due to the difference in the level of external connection of each node, and the relative value is more comparable.

3.3. Preference Level Index. This paper defines $\mathrm{PD}_{i}$ as the preference level index of urban connection, which means cities are most frequently connected with cities at a certain level of $i$ :

$$
\begin{aligned}
M & =\operatorname{Max}\left(\frac{\sum_{j^{l}=1}^{n} \text { Heat }_{i j^{l}}}{\text { Heat_departure }_{i}}\right), \\
\mathrm{PD}_{i} & =f(M) .
\end{aligned}
$$

In the formula, $M$ is the proportion of the sum of travel heat between the city $i$ and the cities within a certain level in the departure heat of city $i$. The sum of the travel heat between the city $i$ and the cities within this level is higher than that between the city $i$ and cities within any levels. $\sum_{j^{l}=1}^{n}$ Heat $_{i j^{l}}$ is the sum of travel heat between city $i$ and cities within the level of $l$ and $l=1,2,3,4,5 n$ is the number of cities within the level of $l$. Heat_departure ${ }_{i}$ is the departure heat of city i. $f(M)$ is the level that $M$ belongs to. $\mathrm{PD}_{i}$ is the preference level of the external contact of node $i$.

3.4. Relative Heat Index. Considering the different levels of external connection of each city, this paper defines the relative heat index to describe the relative connection of cities:

$$
\text { Relative Heat }{ }_{i j}=\frac{\text { Heat }_{i j}}{\text { Heat_departure }_{i}} .
$$

In the formula, Relative Heat ${ }_{i j}$ is the relative travel heat from city $i$ to city $j$, which means the preference degree of city $i$ connecting to city $j$ under the level of the external contact of city $i$. Heat ${ }_{i j}$ is the travel heat between city $i$ and city $j$. Heat_departure ${ }_{i}$ is the departure heat of city $i$, that is, the sum of travel heat from city $i$ to other cities.

\section{Results}

4.1. The General Characteristics of Travel Links between Cities in China. Intercity connections in China are mainly concentrated in Beijing, Shanghai, Chongqing, and a few other cities. The total heat of the top 10 cities accounts for $20.6 \%$ of the total heat of the whole country; that is to say, one fifth of the intercity links in the country are related to these cities. According to the natural fracture point of the total heat of urban travel, Chinese cities are divided into five levels (Figure 1). The level of external connection of cities in the southeast of China is higher than that in the northwest or the northeast. The cities of first four levels 


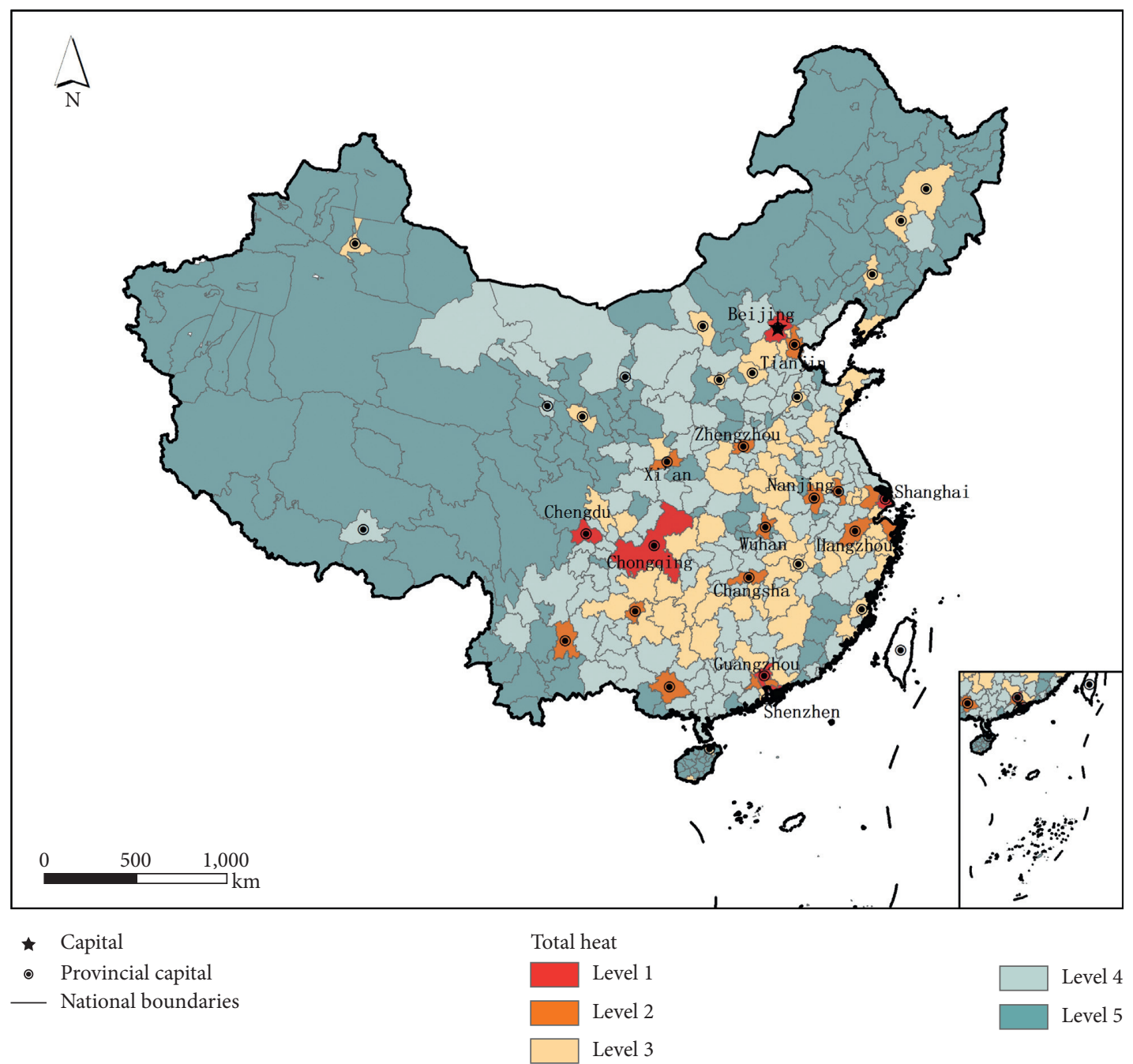

Figure 1: Total heat level of urban travel.

are mainly distributed in the southeast, while the cities in the northwest or the northeast are mainly in the last two levels, among which only Urumqi, Lanzhou, Hohhot, and other provincial capitals have a relative high level of external connection. Beijing, Shanghai, Chongqing, Guangzhou, Chengdu, and Shenzhen belong to the first level. They are located in the four national urban agglomerations of China, Beijing-Tianjin-Hubei urban agglomerations, Yangtze River Delta urban agglomerations, Pearl River Delta urban agglomerations, and Chengdu-Chongqing urban agglomerations. The second level includes some provincial capitals and municipalities directly under the central government, such as Tianjin, Nanjing, and Hangzhou, as well as nonprovincial cities with high economic development level, such as Suzhou and Ningbo. The third level includes other provincial capitals, such as Shijiazhuang and Taiyuan, as well as some nonprovincial capitals which are geographically adjacent to the provincial capitals. The fourth level is mainly formed by other cities in the southeast of China, as well as some provincial capitals in the northwest, such as Lhasa and Xining. Cities in the fifth level are mainly distributed in the northwest and the northeast region of China. The distribution of cities at the third, fourth, and fifth levels are concentrated and contiguous, reflecting that the external contact level of Chinese cities has local characteristics, and the external contact level of a single city is correlated to its neighboring cities to some extent. It is worth noting that this local feature is similar to the concept of "network community" in related research.

The relations between two cities in China also show the characteristics of differentiation in the southeast, northwest, and northeast (Figure 2). The relations between the cities in the southeast are stronger than that in the northwest or northeast. The connection between the first level cities is the most closed, which reflects the rich club characteristics of urban connections. The second, third, and fourth levels of the city link is the connection within cities in the southeast region, as well as between cities in the southeast with the capital city of northwest or northeast China (e.g., Urumqi, Lhasa, and Shenyang), reflecting "Hub-and-Spoke" characteristics of the city network, in which the capital city of northwest and northeast is the "Hub" connecting the 


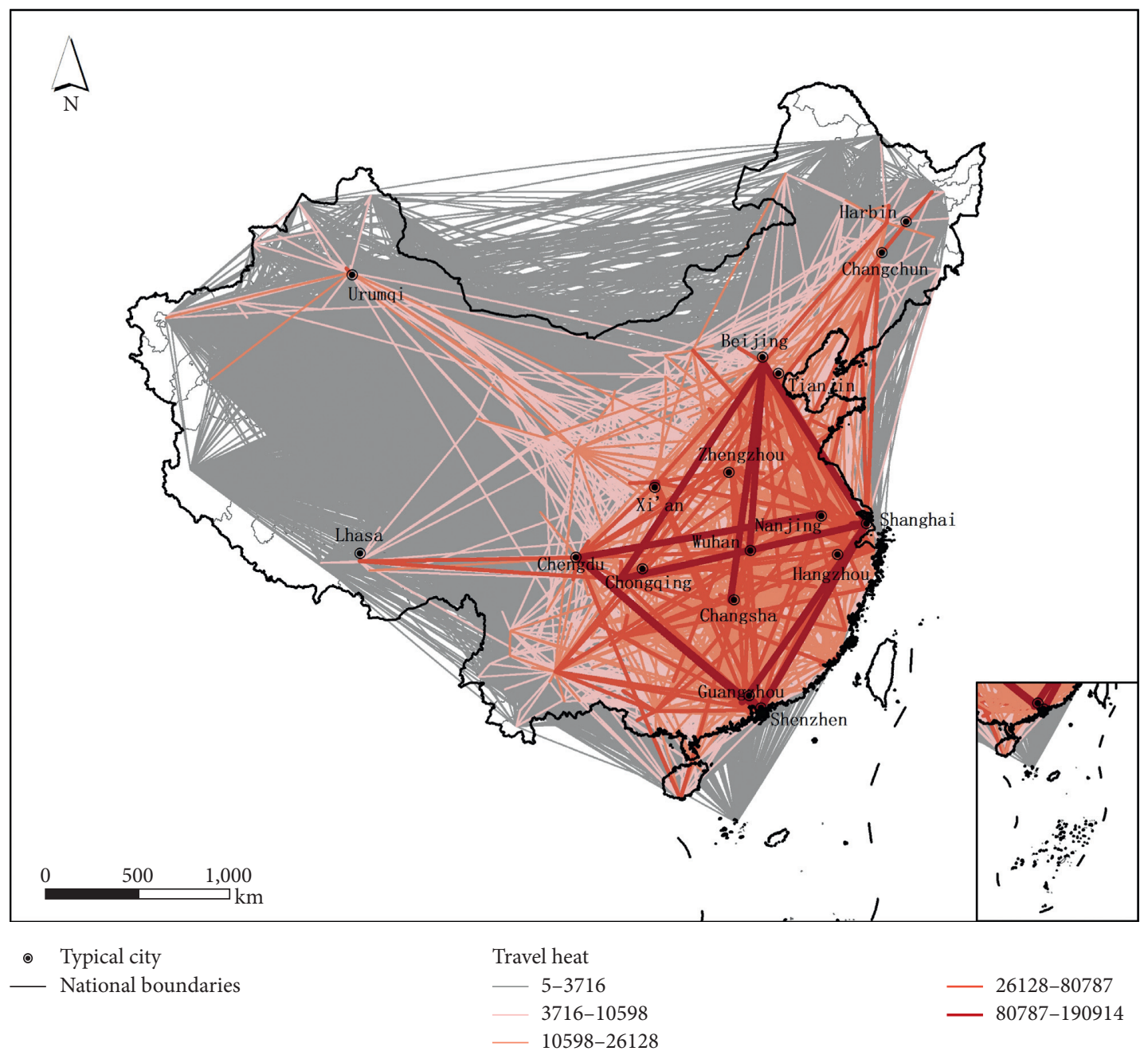

FIGURE 2: Intercity travel heat level.

northwest, northeast, and southeast. On the whole, the intercity relations in China are mainly within the southeast region, while the relations between the southeast region, the northwest region, and the northeast region and the relations within the northwest region are relatively low.

\subsection{The Rich Node Propensity and Hierarchical Proximity of} Intercity Connections. In order to explore whether China's cities prefer to connect with rich nodes (high-level cities) and to measure the degree of this preference quantitatively, this paper calculates the rich node propensity index of each city and finds that the 10 cities with the highest rich node propensity index belong to the third, fourth, or fifth levels (level 3, 4, and 5). Their common feature is that they can be regarded as nonrich nodes, and they are geographically adjacent to and closely related to the rich nodes, which are in the first or second level (level 1 and 2). Therefore, these cities have a high rich node propensity index. For example, Zhoushan in the fifth level is geographically adjacent to Shanghai in the first level, as well as Ningbo and Hangzhou in the second level, which are three cities exactly closely connected with Zhoushan. Close to

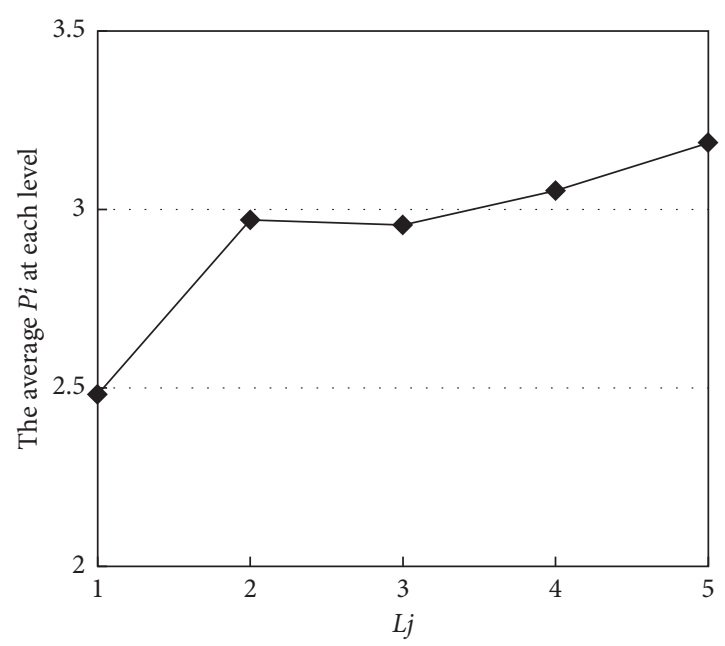

FIGURE 3: Relations between the rich node propensity and city level.

Beijing and Tianjin, Langfang in the third level has the closest relations with them, among which the travel contact with Beijing accounts for $28.27 \%$ of the total 
external connection of Langfang and that with Tianjin accounts for $7.76 \%$.

Although the cities with the highest rich node propensity index belong to nonrich nodes, on the whole, this index has a positive correlation with the city level. Figure 3 shows the relations between the average rich node propensity index of each level and the rich node index. As the city level rises, the more obvious the feature of the rich node is, the stronger the tendency of the city to establish a connection with the rich node is; that is, the preference of the rich node for connecting to the rich node is stronger than that of the nonrich node (Figure 3). The average rich node propensity index of the fifth level is significantly lower than that of the other level; that is to say, the cities with the lowest degree of external connection mostly establish connection with the cities with the same low level of external connection, of which the connection preference with the high-level cities is significantly weaker than that of the other level.

If the connection tendency of nodes to each node is equal, the theoretical value of $P_{i}$ should be 3 . When $P_{i}<3$, it means nodes tend to connect nonrich nodes. When $P_{i}>3$, it means nodes tend to connect rich nodes. Figure 3 shows that the average value of $P_{i}$ of the first and second level are all more than 3, that is, cities in the first and second level (rich nodes) tend to connect the rich nodes, while the average value of $P_{i}$ of the third, fourth, and fifth level are all less than 3 , that is, cities in the third, fourth, and fifth level (nonrich nodes) tend to connect the nonrich nodes.

From the abovementioned analysis, we have got the difference of rich node propensity of external connections of different cities. Next, we explore the preference level of intercity connection, that is, to determine which level is most frequently associated with each city. Through the calculation of $\mathrm{PD}_{i}$, which is the preference level index of each city's external relation, the results show that cities in the first two level have a strong tendency of rich node connection and a tendency of "neighborhood degradation." The preference level of cities in the first level (level 1) is mainly the second and third level of the adjacent level. 33.33\% of cities in the first level have connection preference of the second level, and up to $50 \%$ of cities in the first level have preference of the third level (Figure 4). Although cities in the first level are closely related to cities in the same level, they are more closely related to the provincial capitals and cities with a high level of economic development in the second and third level with a larger base. The preference level of cities in the second level (level 2) is the hierarchically adjacent third and fourth level. $46.67 \%$ of cities of level 2 prefer to connect with cities of level 3, and $53.33 \%$ prefer to connect with cities of level 4 , indicating that some provincial capitals and cities with high level of economic development in the second level tend to be connected with other provincial capitals and cities with relatively high level economic development in the third level. The preference levels of cities in the third and fourth level are mainly cities of level 3 and level 4 . However, cities in the fifth level have the strongest connection with the third, fourth, and fifth level. These results show that cities of level 3,4, and 5 have significant characteristics of the same level and adjacent level connection. It can be seen that cities in the third level have a close relationship with cities of each level. As a bridge between high-level and low-level cities, cities of level 3 constitute the core of the relations between cities in China.

4.3. Geographical Proximity of Intercity Connections. The adjacency effect of urban external connection is not only reflected in the hierarchical proximity but also in the geographical space proximity. Geographic distance plays an important role in population mobility and intercity linkages [46]. Based on the preliminary analysis of the data of travel heat among cities in China, we found that there is, often, a close relationship between cities and their neighboring cities. In order to verify and characterize the impact of geographical proximity on the intercity connection and the difference of that impact on different cities, we carry out this part of the study through the data of the shortest travel time between two cities in China used to represent the geographical distance between cities. We draw the scatter diagram of the travel heat and the shortest travel time of each level of cities (Figure 5). The results show that intercity connection is affected by the distance between cities, that is, geographical proximity. In general, with the decrease in travel time, the relations between cities become closer. Most of China's intercity connections occur within the travel time range of about 7 hours (25000 seconds), and the external connections of cities at all levels peak at the travel time of 2 hours and 5 to 6 hours. With the improvement of the city level, urban external connection is gradually strengthened. When the travel time is 2 hours and 5 to 6 hours, the travel heat of cities in the first and second level is much higher than that of other cities, mainly the connection within high-level cities such as Beijing, Shanghai, Chongqing, Guangzhou, and Shenzhen (Figure 5 inset).

Due to the differences in the degree of external connection between different cities, there may be bias in comparing the differences between different cities by using absolute travel heat. Therefore, we use the relative travel heat Relative Heat ${ }_{i j}$ to further investigate the relations between geographical proximity and intercity connection.

We draw the scatter diagram of the relative travel heat and the shortest travel time of each level of city (Figure 6). The results show that when the travel time is less than 3 hours, cities in the third, fourth, and fifth level have more closely relative external connections, indicating that the short-distance connections between cities mainly occur in the external connection of low-level cities, which are also the main external connection mode of low-level cities. The relative heat of cities in the third, fourth, and fifth levels has increased significantly within a travel time of three hours, even higher than that of high-level cities. Different from the absolute heat, the relative heat of cities in the first and second level has no significant growth trend with the decrease of travel time, showing that the impact of geographical proximity on low-level cities is actually greater than that of high-level cities. The reduction of the geographical distance leads to a significant increase in the external contact strength of low-level cities, and its relative degree of improvement is greater than that of high-level cities. However, due to the 


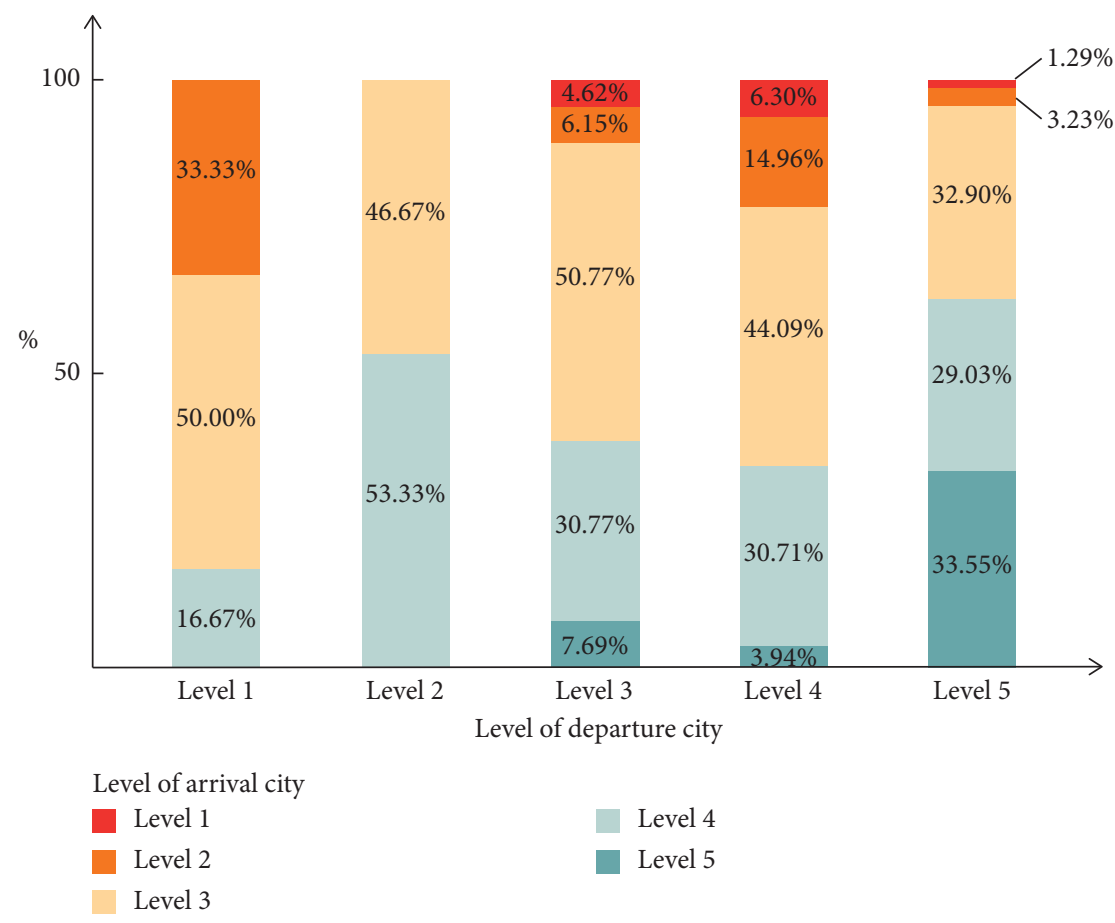

FIGURE 4: Distribution of preference levels of urban external connections.

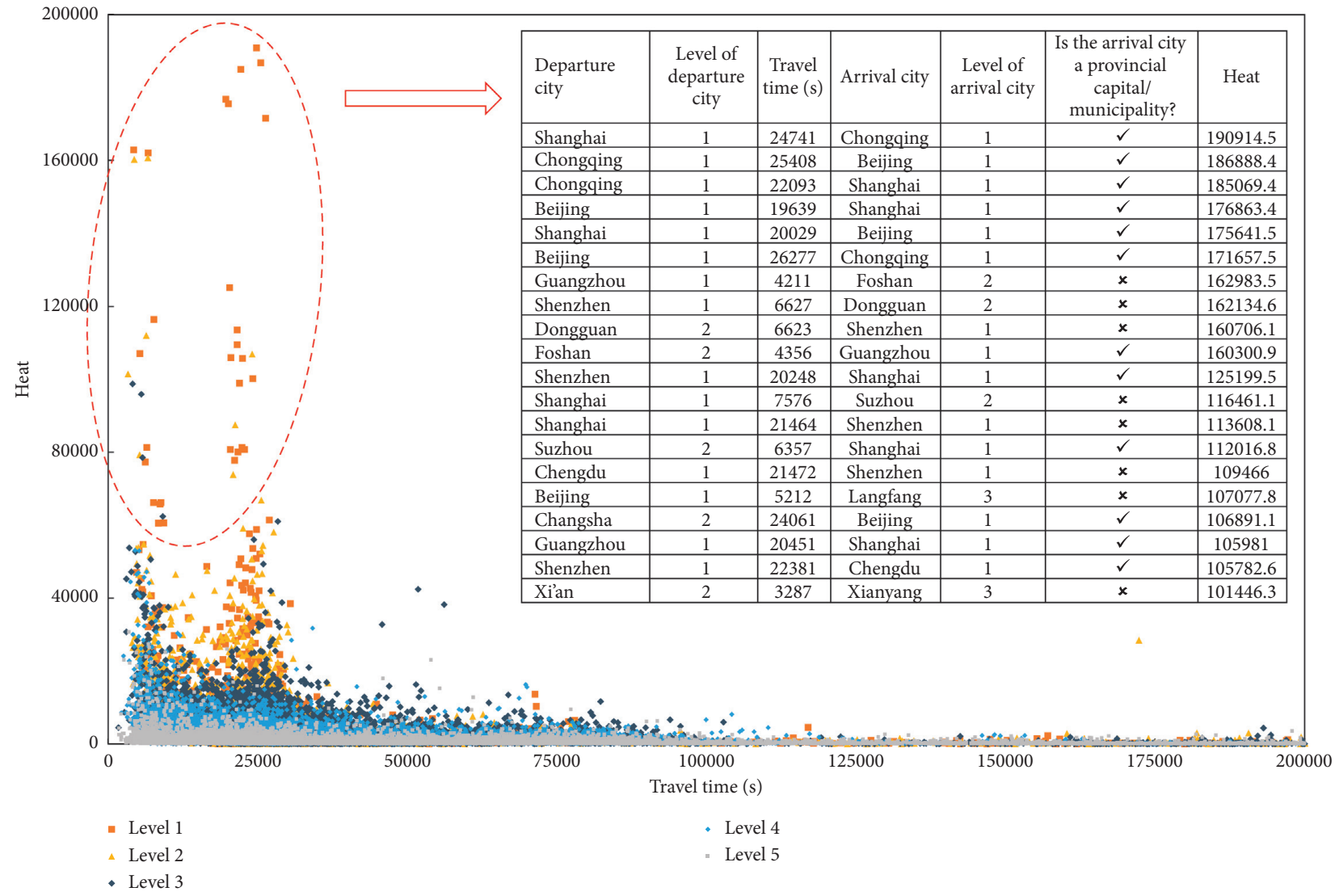

FIGURE 5: Scatter distribution of urban absolute travel heat and shortest travel time. Note. Inset table refers to some intercity links within the scope of an elliptical dotted line. 
stronger external contact of high-level cities, the absolute degree of external contact improvement of high-level cities caused by the reduction of geographical distance is larger than that of low-level cities (Figure 5). Intercity connections with the highest relative heat are between the low-level cities adjacent to the provincial capitals, as well as municipalities directly under the central government and provincial capitals/municipalities in the high level (Figure 6 inset), such as links between Meishan of level 4 and Chengdu of level 1 and between Yuxi of level 5 and Kunming of level 2. This reflects that the high-level provincial capital cities/municipalities directly under the central government have a strong attraction, resulting in a strong "controlling" effect on the geographically adjacent low-level cities. A considerable part of low-level cities have stronger relative external relations than high-level cities, which is due to the strong external contact of high-level cities, so the relative heat between highlevel cities and general cities is relatively low compared to their strong external connection. When the shortest travel time is about $75000 \mathrm{~s}$ (about $20 \mathrm{~h}$ ), the urban external connection of cities of level 5 and level 4 intensity is relative strong, which are mainly linkage between cities of level 5 located in the northwest and cities of level 4 and 3 in the southeast and other cities of level 5 in the northwest, as well as the connection between cities of level 4 and other cities of level 4 and cities in level 3. This reflects that although there is a long distance between some cities, urban connections still exist and have indispensable significance. These cities tend to have the same or similar level, which confirms the hierarchical proximity effect of city connection. This distant but important connection within low-level cities also reflects the complexity of urban connections and urban networks in China.

\section{Discussion on the Characteristics and Modes of the Intercity Connection}

Based on the abovementioned analysis, this paper summarizes the characteristics and modes of the intercity connection in China. The relations between China's cities mainly have club characteristics, geographical proximity, hierarchical proximity effect, and also a significant spatial distance threshold effect.

5.1. Club Characteristics and the Geographical Proximity Effect. The connection between cities in China has club characteristics (Figure 7). The rich nodes (high-level cities) tend to connect with rich nodes, consistent with the conclusions of the work of Opsahl [42] and Wei [30]. With the improvement of the city level, the tendency of connecting rich nodes is stronger. Nonrich nodes (low-level cities) tend to choose to connect with nonrich nodes. With the decrease of the city level, the willingness of cities to connect with nonrich nodes is stronger. The rich node has a strong attraction; thus, the nonrich nodes in the geographical vicinity have a close relationship with it, and this relationship occupies a considerable proportion in the external relations of the nonrich nodes adjacent to rich nodes.
Rich nodes and nonrich nodes are affected by geographical proximity. The shorter the geographical distance is, the closer the relations between cities is. By comparing the change rules of absolute heat and relative heat over travel time (Figure 5 vs. 6), it is found that as travel time shortens, the higher the city level is, that is, the more significant the rich node feature is, the more obvious the increase of absolute heat of the city is. However, the relative heat has the opposite rule. With the shortening of travel time, the lower the city level is, that is, the more significant the characteristic of nonrich nodes is, the more significant the increase of the relative heat of the city is. This indicates that rich nodes and nonrich nodes are affected differently by the geographical proximity effect. The shortening of geographical distance mainly improves the absolute external contact intensity of rich nodes. Because of strong total external contact intensity of rich nodes, such improvement takes a small proportion in the total external contact intensity of rich nodes, so the relative external contact (i.e., relative heat) of rich nodes is not improved much. The shortening of geographical distance mainly increases the relative external connection strength of nonrich nodes. The total external connection strength of nonrich nodes is low, and the increase in the intensity of connections brought about by the geographical proximity occupies a relative large proportion of the total contact. Therefore, although the absolute external connection of nonrich nodes is not significantly improved by distance shortening, its relative external connection is improved greatly, even greater than the degree of improvement of rich nodes. Nonrich nodes (low-level cities) are actually more affected by geographical proximity than rich nodes (high-level cities). In addition, the short-distance connection mostly occurs in the external connection of nonrich nodes and also is the main external connection mode of nonrich nodes. On the other hand, although the geographical proximity effect has a profound impact on the connections between Chinese cities, there are still connections between lower-level cities far apart, which are driven by other factors and of indispensable importance. In fact, this "remote" connection exists widely in the real network [47]. Sorenson [48] found that it included many "distant" ties between parties that vary on one or more social dimensions by studying the venture capital network in America.

5.2. Hierarchical Proximity Effect. The connection between cities in China has a hierarchical proximity effect (Figure 8). The cities with hierarchical proximity are closely related. Cities in the first and second levels have the characteristics of the "neighborhood degradation" connection, which shows that cities of level 1 prefer to connect with cities in the second and third levels, and cities of level 2 prefer to connect with cities in the third and fourth levels. Cities in the third, fourth, and fifth levels have characteristics of the same level and adjacent level connection, among which cities of level 3 and level 4 are closely connected with cities of level 3 and level 4 , and cities of level 5 prefer to connect with cities of level 3, 4, and 5. The hierarchical proximity effect causes cities of level 3 to both form a close relationship with the high-level cities 


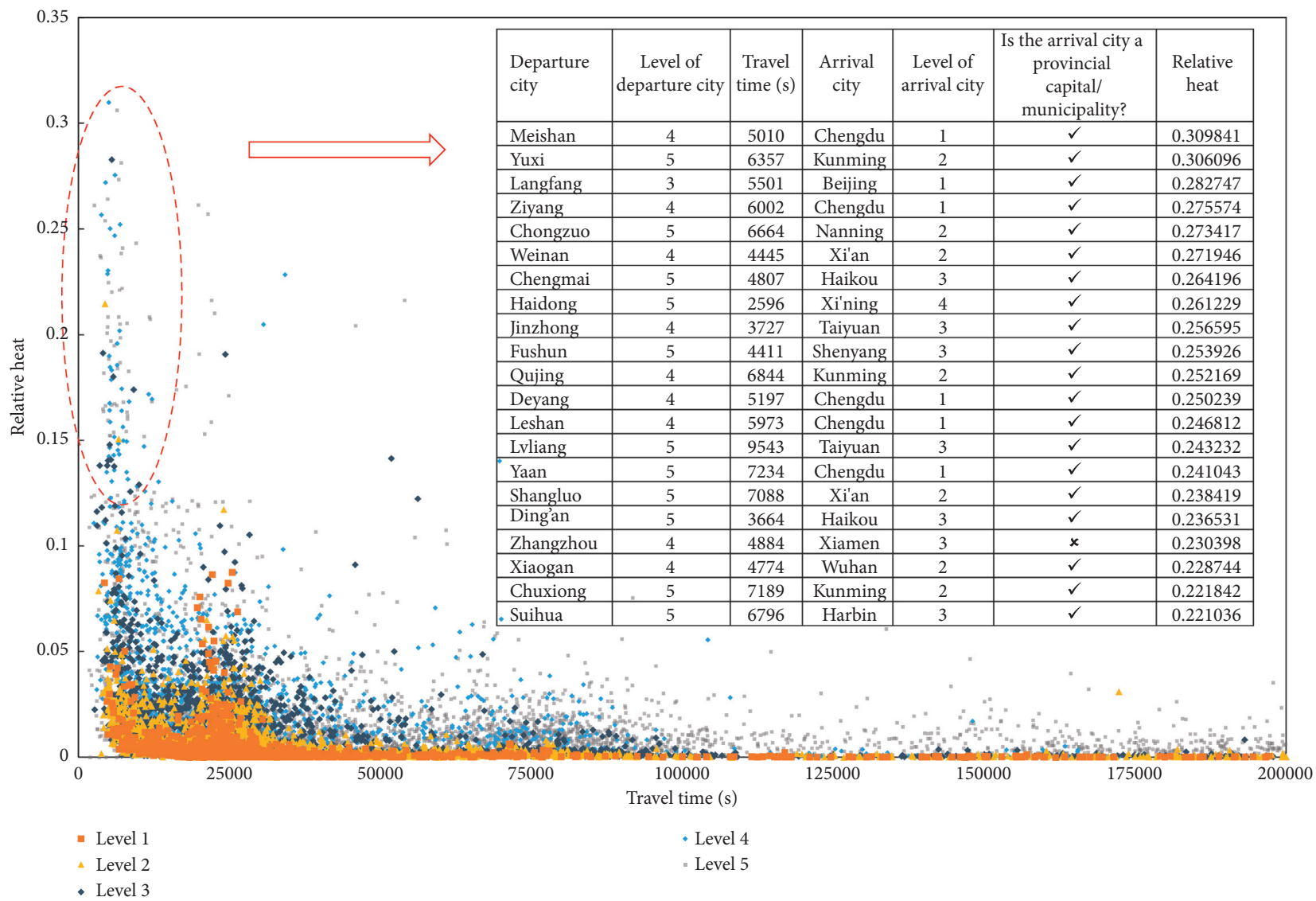

Figure 6: Scatter distribution of urban relative travel heat and shortest travel time. Note. Inset table refers to some intercity relative links within the scope of an elliptical dotted line.

and the low-level cities, playing an important role as a bridge. On the other hand, the hierarchical proximity effect of intercity connections reflects "hierarchical isolation," that is, cities with relatively far hierarchical distance have sparse connections. For example, there are weak connections between cities of level 5 and cities of level 1. The theory of "hierarchical diffusion" [49] reflects a degree of "hierarchical isolation". Hierarchical diffusion reflects the order of diffusion, which is seldom manifested as one step spread from a high-level city to a low-level city. This order highlights the hierarchical isolation between the high-level city and lowlevel city.

The hierarchical proximity effect of city connections is similar to that of club characteristics. Cities tend to establish relationships with cities with similar attributes; for example, provincial capitals tend to connect with other provincial capitals of the same level. The hierarchical proximity effect of urban linkages can also be found in the existing literature. Meijers [50] shows that it appears that these club-type networks provide much better conditions for achieving horizontal synergy than ever before. The reason for the hierarchical proximity effect may be that horizontal linkages have lower "communication costs," including similar economic size, population size, and institutional environment.

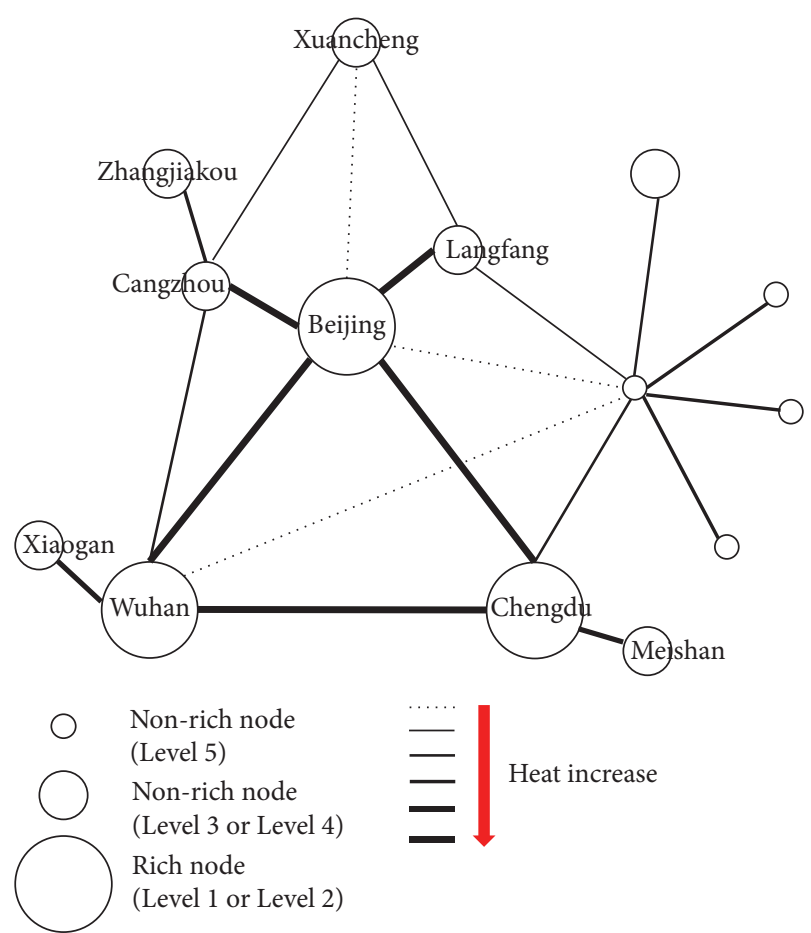

Figure 7: Club characteristics and the geographical proximity effect of intercity connections. 


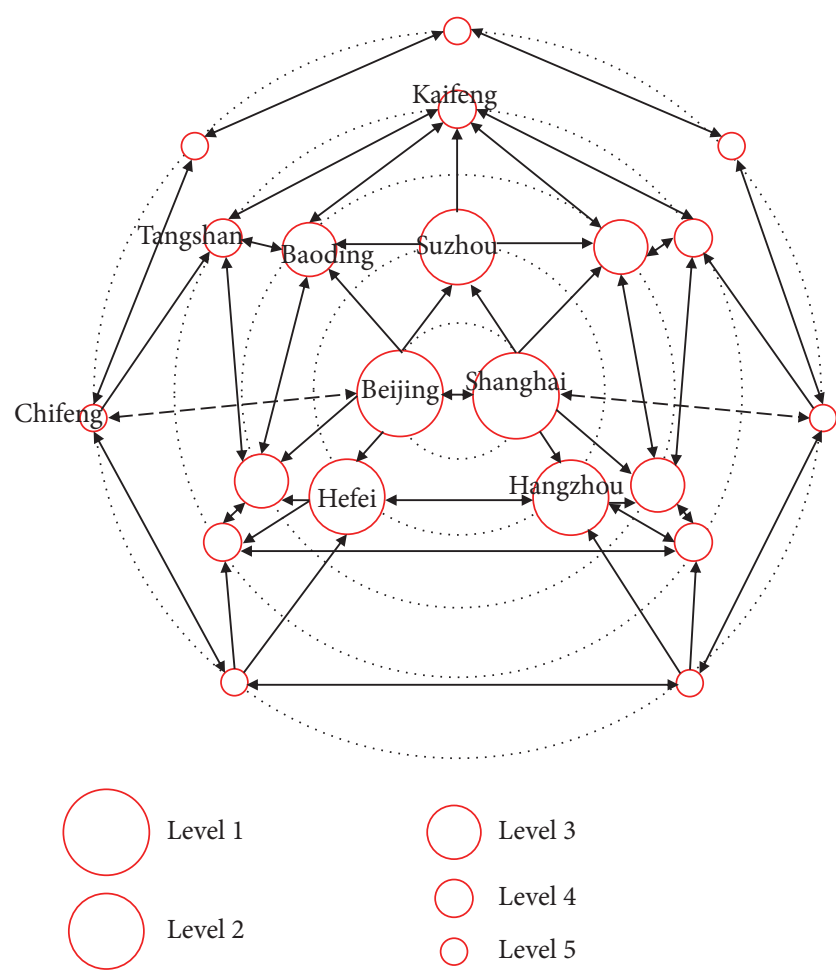

FIGURE 8: Hierarchical proximity effect of intercity connections.

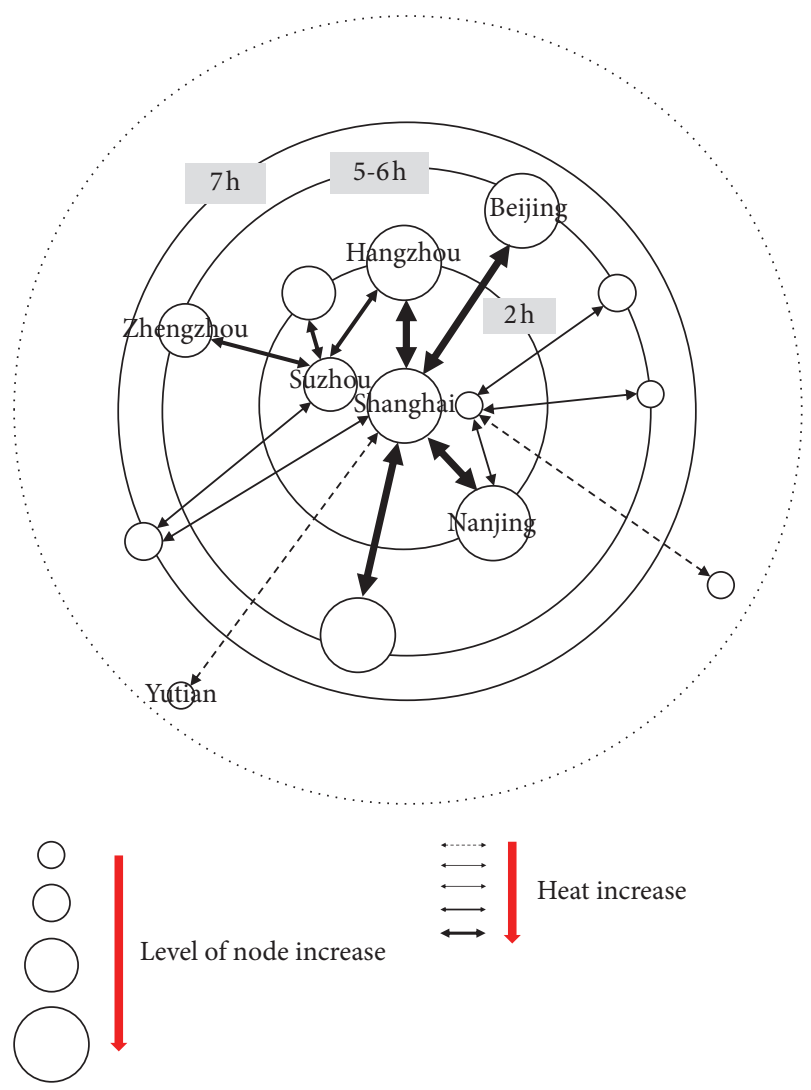

FIGURE 9: Spatial distance threshold effect of intercity connections.

5.3. Spatial Distance Threshold Effect. The shortest travel time between most cities in China is within 7 hours, and when the shortest travel time is more than 7 hours, the intercity connection becomes weak (Figure 9). Two hours and 5-6 hours are the "golden distance" of intercity connection. When the shortest travel time between two cities is 2 hours or 5-6 hours, the intercity connection is often close. The "golden distance" of $2 \mathrm{~h}$ and $5-6 \mathrm{~h}$ is obviously reflected in the connection between high-level cities. The connection between high-level cities is far stronger than that between other cities when the shortest travel time is 2 hours or 5-6 hours. The most significant "golden distance" of low-level cities is $3 \mathrm{~h}$. Within $3 \mathrm{~h}$ of travel time, the external contact of low-level cities is significantly improved, which mainly occurs between low-level cities adjacent to high-level cities and high-level cities. There is a difference in the "golden distance" between high-level and low-level cities. Despite these two kinds of cities are subjected to the constraints of geographical distance, high-level cities are less affected and have strong willingness to connect with high-level cities that are far apart (5-6h), while the low-level cities are more willing to associate with high-level cities in close proximity (within $3 \mathrm{~h}$ ).

\section{Conclusions}

In the recent years, the relations between cities in China are increasingly close, so it is of great significance to explore the characteristics of intercity connections. From the perspective of intercity population travel, the characteristics of intercity connections are investigated with the big data of Tencent's population migration heat.

The research has the following results. First, China's intercity links are mainly concentrated in Beijing, Shanghai, Chongqing, and a few other rich node cities. The connection between cities in China has club characteristics; that is, the rich nodes (high-level cities) tend to connect with rich nodes, consistent with the conclusions of work of Opsahl [42] and Wei [30]. The higher the city level is, the tendency of connecting rich nodes is stronger. The nonrich nodes tend to connect with the nonrich nodes, and the lower the city level is, the stronger the preference of connecting nonrich nodes is. Second, the connection between cities in China has a hierarchical proximity effect. For example, cities in the first (second) level are mainly connected with cities in the second and third (third and fourth) levels adjacent to the level. Cities in the third and fourth level are mainly connected with cities in the third and fourth level. At the same time, cities with relatively far hierarchical distance have sparse connections, that is, "hierarchical isolation," which is similar to the law revealed by hierarchical diffusion [49]. Hierarchical diffusion reflects the order of diffusion, which is seldom manifested as one step spread from a high-level city to a low-level city. This order highlights the hierarchical isolation between the high-level city and low-level city. Third, the connection between cities in China has a geographical proximity effect. On the whole, with the geographical distance between cities shortened, the relations between cities became closer. The impact of geographical proximity on low-level cities is greater than that on high-level cities. The short-distance urban connection is often found in the external connection of low-level cities and is also the main external connection 
mode of low-level cities. The high-level provincial capital cities and municipalities directly under the central government have strong attraction, coupling with the influence of geographical proximity factors, resulting in very close relations between low-level cities that are geographically adjacent to the high-level cities and high-level cities. This relation accounts for a large proportion of the external relations of such low-level cities. Fourth, intercity connections also have the characteristics of the spatial distance threshold effect. When the shortest travel time between cities is 2 hours or 5-6 hours, the relations between cities are close. However, the "golden distance" of external contact between high-level cities and low-level cities is different because rich nodes and nonrich nodes are affected differently by the geographical proximity effect. In addition, China's urban network also has some long-distance connections within low-level cities, which are of great significance and widely exist in the real network [47, 48]. The external connection mode of high-level cities is to connect with high-level cities with the same level or hierarchically adjacent to them and also has strong attraction to low-level cities with geographical proximity. The low-level cities are closely related to the high-level cities with geographical proximity, as well as other cities with geographical proximity or hierarchical proximity.

This paper holds that hierarchical proximity and geographical proximity are important factors affecting intercity relations, and this conclusion can be applied to regional planning and urban policy. Urban external contacts not only bring vitality to urban development but also bring risks, such as infectious diseases. Based on the fact that urban linkages are affected by hierarchical proximity and geographical proximity, the mechanisms of the spread of infectious diseases between regions and within regions can be explained accordingly, and the risk of infectious diseases in regions/cities can be more accurately predicted and assessed. In the event of an infectious disease crisis, a focus should be placed on the risk status of cities hierarchically or geographically adjacent to the city to predict the risk of the city. In addition, as low-level cities are strongly influenced by the geographical proximity effect, if we want to focus on the development of small or medium-sized cities, we should consider strengthening the leading role of large cities (higher-level cities) that are geographically adjacent to small- or medium-sized cities. The effect of geographical proximity on high-level cities is relatively weak, the influence of hierarchical proximity on high-level cities should be fully considered. If we want to focus on developing big cities (high-level cities), we should strengthen the cooperation within high-level cities.

This paper comprehensively investigates the external connection characteristics of rich nodes and nonrich nodes, improves the deficiencies of previous studies that only focus on rich nodes and the phenomenon of "rich club", and deepens the cognition of "rich club" through the quantitative analysis of the connection preference of cities. On the other hand, it is helpful to further understand the mechanism behind the intercity connection by studying the difference of the effect of geographical proximity on external connection of different cities. By observing Chinese cases, this study finds out the similarity and proximity laws of urban connections and puts forward the club characteristics, hierarchical proximity effect, and geographical proximity effect of intercity connections. In the past, the research consensus was "hierarchical diffusion," that is, the step-like sprawl from high-level cities to low-level cities, emphasizing the hierarchical characteristics of urban networks. In this paper, the hierarchical proximity effect of urban connections is found, that is, both cities of high and low levels tend to establish relationships with cities of similar or the same level, emphasizing the flattening of urban networks. In addition, this study proposes the spatial distance threshold effect characteristic of an urban connection, which enhances the attention to the spatial nature of the urban connection and urban network. This study mainly focuses on two dominant factors, rich node tendency and geographical proximity of urban external connections. However, the source and vitality of intercity links are people, and the subjective will of people is another important factor of intercity links and urban networks, which is not discussed in this paper. In future studies, we will pay more attention to people's microneeds so as to further explore and understand the characteristics and operation mechanism of urban linkages.

\section{Data Availability}

Tencent's population migration heat data are obtained from the location big data released by the Tencent company, which come from https://heat.qq.com/. The shortest travel time data between cities are obtained from the Baidu map product released by the Baidu company, which come from https://map.baidu.com/.

\section{Conflicts of Interest}

The authors declare that no conflicts of interest exist with regard to the publication of this paper.

\section{Acknowledgments}

This research was supported by the National Natural Science Foundation of China (grant nos. 41822104, 41771133, and 41671125), the Chinese Academy of Sciences Basic Frontier Science Research Program from 0 to 1 Original Innovation Project (no. ZDBS-LY-DQC005), the Strategic Priority Research Program of the Chinese Academy of Sciences (grant no. XDA23100301), and the Youth Innovation Promotion Association of the Chinese Academy of Science (grant no. 2017072).

\section{References}

[1] M. Chen, Y. Gong, D. Lu, and C. Ye, "Build a people-oriented urbanization: China's new-type urbanization dream and Anhui model," Land Use Policy, vol. 80, pp. 1-9, 2019.

[2] M. Chen, W. Liu, D. Lu, H. Chen et al., "Progress of China's new-type urbanization construction since 2014: a preliminary assessment," Cities, vol. 78, pp. 180-193, 2018. 
[3] H. Li, Y. D. Wei, and Y. Zhou, "Spatiotemporal analysis of land development in transitional China," Habitat International, vol. 67, pp. 79-95, 2017.

[4] C. Chen, T. D’Alfonso, H. Guo, and C. Jiang, "Graph theoretical analysis of the Chinese high-speed rail network over time," Research in Transportation Economics, vol. 72, pp. 3-14, 2018.

[5] M. Chen, L. Liang, Z. Wang, W. Zhang et al., "Geographical thoughts on the relationship between 'Beautiful China' and land spatial planning," Journal of Geographical Sciences, vol. 30, no. 5, pp. 705-723, 2020.

[6] J. Pan and J. Lai, "Spatial pattern of population mobility among cities in China: case study of the National Day plus Mid-Autumn Festival based on Tencent migration data," Cities, vol. 94, pp. 55-69, 2019.

[7] J. Pan and J. Lai, "Research on spatial pattern of population mobility among cities: a case study of "Tencent Migration" big data in "National Day-Mid-Autumn Festival" vacation," Geographical Research, vol. 38, pp. 1678-1693, 2019.

[8] R. Camagni, S. Stabilini, and L. Diappi, "City networks in the lombardy region: an analysis in terms of communication flows," Flux, vol. 10, no. 15, pp. 37-50, 1994.

[9] L. M. Ronald and O. W. James, "The flow of information in a global economy: the role of the American urban system in 1990," Annals of the Association of American Geographers, vol. 84, no. 1, pp. 87-107, 1994.

[10] B. Derudder and F. Witlox, "Mapping world city networks through airline flows: context, relevance, and problems," Journal of Transport Geography, vol. 16, no. 5, pp. 305-312, 2008.

[11] N. Limtanakool, T. Schwanen, and M. Dijst, "Developments in the Dutch urban system on the basis of flows," Regional Studies, vol. 43, no. 2, pp. 179-196, 2009.

[12] H. Matsumoto, "International urban systems and air passenger and cargo flows: some calculations," Journal of Air Transport Management, vol. 10, no. 4, pp. 239-247, 2004.

[13] W.-S. Jung, F. Wang, and H. E. Stanley, "Gravity model in the Korean highway," Europhysics Letters, vol. 81, no. 4, Article ID 48005, 2008.

[14] H.-S. Lee, "The networkability of cities in the international air passenger flows 1992-2004," Journal of Transport Geography, vol. 17, no. 3, pp. 166-175, 2009.

[15] A. S. Alderson and J. Beckfield, "Power and position in the world city system," American Journal of Sociology, vol. 109, no. 4, pp. 811-851, 2004.

[16] A. S. Alderson, J. Beckfield, and J. Sprague-Jones, "Inter-city Relations and Globalization: The Evolution of the Global Urban Hierarchy, 1981-2007," Urban Studies, vol. 47, no. 9, pp. 1899-1923, 2010.

[17] J. Osorio-Arjona and J. C. García-Palomares, "Social media and urban mobility: using twitter to calculate home-work travel matrices," Cities, vol. 89, pp. 268-280, 2019.

[18] H. T. Ma, C. L. Fang, S. N. Lin et al., "Hierarchy, clusters, and spatial differences in Chinese inter-city networks constructed by scientific collaborators," Journal of Geographical Sciences, vol. 12, pp. 1793-1809, 2018.

[19] L. Van Der Laan, "Changing urban systems: an empirical analysis at two spatial levels," Regional Studies, vol. 32, no. 3, pp. 235-247, 1998.

[20] D. Burgalassi, "Defining and Measuring Polycentric Regions: The Case of Tuscany," Mpra Paper, Article ID 25880, 38 pages, 2010.

[21] D. Karemera, V. I. Oguledo, and B. Davis, "A gravity model analysis of international migration to North America," Applied Economics, vol. 32, no. 13, pp. 1745-1755, 2000.
[22] M. Barthélemy, "Spatial networks," Physics Reports, vol. 499, no. 1-3, pp. 1-101, 2011.

[23] M. Chen, C. Ye, D. Lu et al., "Cognition and construction of the theoretical connotations of new urbanization with Chinese characteristics," Journal of Geographical Sciences, vol. 29, no. 10, pp. 1681-1698, 2019.

[24] J. Yang, H. You, Y. Zhang, and C. Jin, "Research progress on human settlements: from traditional data to big data+," Progress in Geography, vol. 39, no. 1, pp. 166-176, 2020.

[25] J. Li, Q. Ye, X. Deng et al., "Spatial-temporal analysis on spring festival travel rush in China based on multisource big data," Sustainability, vol. 8, p. 1184, 2016.

[26] J. Xu, A. Li, D. Li, Y. Liu et al., "Difference of urban development in China from the perspective of passenger transport around Spring Festival," Applied Geography, vol. 87, pp. 8596, 2017.

[27] J. Yang, D. Yi, J. Liu, Y. Liu et al., "Spatiotemporal change characteristics of nodes' heterogeneity in the directed and weighted spatial interaction networks: case study within the sixth ring road of Beijing, China," Sustainability, vol. 11, p. 6359, 2019.

[28] W. Zhang, B. Derudder, J. Wang et al., "Using location-based social media to chart the patterns of people moving between cities: the case of weibo-users in the Yangtze River Delta," Journal of Urban Technology, vol. 23, no. 3, pp. 91-111, 2016.

[29] J. Yang, A. Guo, X. Li, and T. Huang, "Study of the impact of a high-speed railway opening on China's accessibility pattern and spatial equality," Sustainability, vol. 10, no. 8, p. 2943, 2018.

[30] Y. Wei, W. Song, C. Xiu, and Z. Zhao, "The rich-club phenomenon of China's population flow network during the country's spring festival," Applied Geography, vol. 96, pp. 77-85, 2018.

[31] J. Park, L. B. Wood, E. Jing et al., "Global labor flow network reveals the hierarchical organization and dynamics of geo-industrial clusters," Nature Communications, vol. 10, p. 3449, 2019.

[32] W. Ke, W. Chen, and Z. Yu, "Uncovering spatial structures of regional city networks from expressway traffic flow data: a case study from jiangsu province, China," Sustainability, vol. 9, p. 1541, 2017.

[33] S. Wickramasinghe, O. Onyerikwu, J. Sun, and D. BenAvraham, "Modeling spatial social complex networks for dynamical processes," Complexity, vol. 2018, Article ID 1428719, 12 pages, 2018.

[34] M. De Domenico, V. Nicosia, A. Arenas, and V. Latora, "Structural reducibility of multilayer networks," Nature Communications, vol. 6, p. 6864, 2015.

[35] R. Guimera and L. A. N. Amaral, "Modeling the world-wide airport network," European Physical Journal B, vol. 38, no. 2, pp. 381-385, 2004.

[36] F. John, "The world city hypothesis," Development \& Change, vol. 17, pp. 69-83, 1986.

[37] Z. Xu and R. Harriss, "Exploring the structure of the U.S. intercity passenger air transportation network: a weighted complex network approach," GeoJournal, vol. 73, no. 2, pp. 87-102, 2008.

[38] D. Brockmann, L. Hufnagel, and T. Geisel, "The scaling laws of human travel," Nature, vol. 439, no. 7075, pp. 462-465, 2006.

[39] X. Yan, W. Wang, Z. Gao, and Y. Lai, "Universal model of individual and population mobility on diverse spatial scales," Nature Communications, vol. 8, Article ID 1639, 2017.

[40] F. Xie, J. Lin, and W. Cui, "Exploring express delivery networks in China based on complex network theory," Complexity, vol. 21, no. 2, pp. 166-179, 2015. 
[41] J. Alstott, P. Panzarasa, M. Rubinov et al., "A unifying framework for measuring weighted rich clubs," Scientific Reports, vol. 4, p. 7258, 2014.

[42] T. Opsahl, V. Colizza, P. Panzarasa, and J. J. Ramasco, "Prominence and control:The weighted rich-club effect," Physical Review Letters, vol. 101, no. 16, pp. 3958-3964, 2008.

[43] V. Colizza, A. Flammini, M. A. Serrano, and A. Vespignani, "Detecting rich-club ordering in complex networks," Nature Physics, vol. 2, no. 2, pp. 110-115, 2006.

[44] D. Smilkov and L. Kocarev, "Rich-club and page-club coefficients for directed graphs," Physica a Statistical Mechanics \& Its Applications, vol. 389, no. 11, pp. 2290-2299, 2011.

[45] S. Zhou and R. J. Mondragón, "The rich-club phenomenon in the Internet topology," IEEE Communications Letters, vol. 8, no. 3, pp. 180-182, 2004.

[46] L. Gadar, Z. T. Kosztyan, and J. Abonyi, "The settlement structure is reflected in personal investments: distance-dependent network modularity-based measurement of regional attractiveness," Complexity, vol. 2018, Article ID 1306704, 16 pages, 2018.

[47] D. J. Watts and S. H. Strogatz, "Collective dynamics of "smallworld" networks," Nature, vol. 393, no. 6684, pp. 440-442, 1998.

[48] O. Sorenson and T. E. Stuart, "Bringing the context back in: settings and the search for syndicate partners in venture capital investment networks," Administrative Science Quarterly, vol. 53, no. 2, pp. 266-294, 2008.

[49] J. C. Hudson, "Diffusion in a central place system," Geographical Analysis, vol. 1, no. 1, pp. 45-58, 1969.

[50] E. Meijers, "Polycentric urban regions and the quest for synergy: is a network of cities more than the sum of the parts?" Urban Studies, vol. 42, no. 4, pp. 765-781, 2005. 